\title{
Novel shape evolution in exotic Ni isotopes and configuration-dependent shell structure
}

\author{
Yusuke Tsunoda, ${ }^{1}$ Takaharu Otsuka, ${ }^{1,2,3}$ Noritaka Shimizu, ${ }^{2}$ Michio Honma, ${ }^{4}$ and Yutaka Utsuno ${ }^{5}$ \\ ${ }^{1}$ Department of Physics, University of Tokyo, Hongo, Bunkyo-ku, Tokyo 113-0033, Japan \\ ${ }^{2}$ Center for Nuclear Study, University of Tokyo, Hongo, Bunkyo-ku, Tokyo 113-0033, Japan \\ ${ }^{3}$ National Superconducting Cyclotron Laboratory, Michigan State University, East Lansing, Michigan 48824, USA \\ ${ }^{4}$ Center for Mathematical Sciences, University of Aizu, Ikki-machi, Aizu-Wakamatsu, Fukushima 965-8580, Japan \\ ${ }^{5}$ Advanced Science Research Center, Japan Atomic Energy Agency, Tokai, Ibaraki 319-1195, Japan \\ (Received 19 September 2013; revised manuscript received 25 November 2013; published 17 March 2014)
}

\begin{abstract}
The shapes of neutron-rich exotic Ni isotopes are studied. Large-scale shell model calculations are performed by the advanced Monte Carlo shell model (MCSM) for the $p f-g_{9 / 2}-d_{5 / 2}$ model space. Experimental energy levels are reproduced well by a single fixed Hamiltonian. Intrinsic shapes are analyzed for MCSM eigenstates. Intriguing interplays among spherical, oblate, prolate, and $\gamma$-unstable shapes are seen, including shape fluctuations, E(5)-like situations, the magicity of doubly magic ${ }^{56,68,78} \mathrm{Ni}$, and the coexistence of spherical and strongly deformed shapes. Regarding the last point, strong deformation and change of shell structure can take place simultaneously, being driven by the combination of the tensor force and changes of major configurations within the same nucleus.
\end{abstract}

DOI: 10.1103/PhysRevC.89.031301

PACS number(s): 21.10.-k, 21.60.Cs, 21.60.Fw, 27.50.+e

Atomic nuclei exhibit simple and robust regularities in their structure comprised of $Z$ protons and $N$ neutrons. A very early example is the (spherical) magic numbers conceived by Mayer and Jensen [1]. These magic numbers dominate lowenergy dynamics of stable nuclei and their neighbors on the Segré chart. Another basic feature is nuclear shape, which has been one of the central issues of nuclear physics since the work of Rainwater [2] and Bohr and Mottelson [3]. The shape varies as $Z$ or $N$ changes in such a way that it tends to be spherical near magic numbers, while it becomes more deformed towards the middle of the shell. Recent theoretical and experimental studies on exotic nuclei with unbalanced $Z$ and $N$ cast challenges to these pictures. Even magic numbers are not an exception: the changes of the shell structure due to nuclear forces, referred to as shell evolution [4], have been seen, including disappearance of traditional magic numbers and appearance of new ones. A recent example is the discovery of the $N=34$ magic number [5], after its prediction a decade ago [6], while many other cases have been discussed [4,7-9].

It is, thus, of much interest to explore shapes of exotic nuclei and to look for relations to the shell evolution. In this Rapid Communication, we report results of state-of-the-art large-scale shell-model calculations for a wide range of $\mathrm{Ni}$ isotopes, focusing on these points. While the ground state turns out to be basically spherical, a strongly prolate deformed band appears at low excitation energy in some nuclei, similar to shape coexistence, known in other nuclei over decades, e.g., [10-12]. We shall present that the shell structure, for instance, the spin-orbit splitting, can be varied significantly between such spherical and deformed states by a combined effect of different major configurations and the nuclear forces, particularly the proton-neutron tensor force. This phenomenon occurs within the same nucleus, and thereby is not described as shell evolution in the conventional sense. However, to discuss the basic underlying physics in a unified way, this phenomenon will be called Type II shell evolution, while the shell evolution by the change of $N$ or $Z$ will be referred to as Type I. We shall discuss other interesting features, e.g., varying appearance of magicity in ${ }^{56,68,78} \mathrm{Ni}$, shape fluctuations including $\gamma$ instability, and the $\mathrm{E}(5)$-like case [13].

We discuss, in this Rapid Communication, the structure of $\mathrm{Ni}$ isotopes of even $N=28-50$, utilizing results of the advanced Monte Carlo shell model (MCSM) calculation [14-16] run on the $K$ computer for $\sim 2 \times 10^{10}$ core seconds in total. The model space consists of the full $p f$ shell, $0 g_{9 / 2}$ and $1 d_{5 / 2}$ orbits for both protons and neutrons. There is no truncation within this space, as an advantage of MCSM. The Hamiltonian is based on the A3DA Hamiltonian with minor revisions $[14,17]$. The spurious center-of-mass motion is removed by the Lawson method [18].

Figure 1 shows yrast and yrare levels by the present calculation compared to experiment [19-21]. Systematic behaviors are visible in experimental yrast levels as well as $J^{\pi}=0_{2}^{+}$and $2_{2}^{+}$yrare levels, with a remarkable agreement to the theoretical trends. Such good agreement has been obtained with a single fixed Hamiltonian, and suggests that the structure of $\mathrm{Ni}$ isotopes can be studied with it. The $B\left(E 2 ; 0_{1}^{+} \rightarrow 2_{1}^{+}\right)$values with neutron and proton effective charges, 0.5 and 1.5 , respectively, are shown in Fig. 1 compared to experiment [22] with certain discrepancies for heavier isotopes, where uncertainties are larger and $\left(p, p^{\prime}\right)$ data are converted $(N=46)[23,24]$. A more systematic comparison with precise data is desired. Relevant shell-model calculations have been reported [25,26]. In particular, those of [26] are a remarkable achievement of the large-scale conventional shellmodel approach, with good agreement to experiment. Many experimental data are yet to be obtained. For instance, the $0_{2}^{+}$ level of ${ }^{68} \mathrm{Ni}$ has only recently been corrected [20,21]. The primary objective of this Rapid Communication is to predict novel systematic change of band structures in ${ }^{68-78} \mathrm{Ni}$ isotopes and to present the under-lying robust mechanisms for them.

We show in Fig. 2 a more detailed level scheme for ${ }^{68} \mathrm{Ni}$, including negative-parity states. This nucleus has attracted much attention [20,21,25-34] from both theoretical and experimental sides. The positive-parity levels are classified according to their shape categories: spherical, oblate, and 


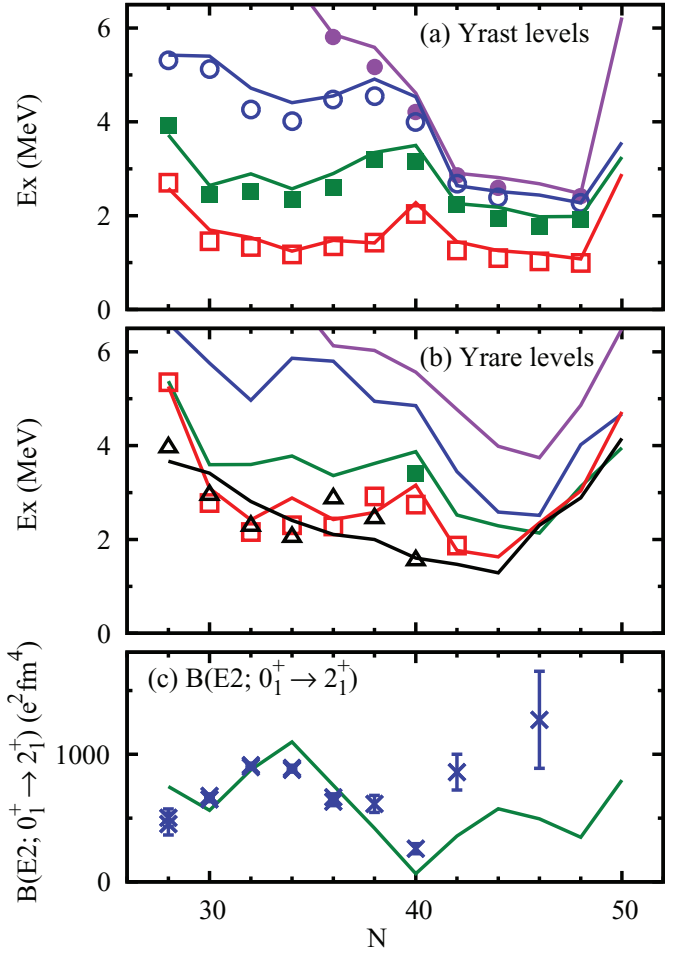

FIG. 1. (Color online) Energy levels for (a) yrast and (b) yrare states of $\mathrm{Ni}$ isotopes with even $N$. Symbols are experimental data for $J^{\pi}=0^{+}$(black triangles), $2^{+}$(open red squares), $4^{+}$(green filled squares), $6^{+}$(open blue circles), and $8^{+}$(filled purple circles) [19-21]. Lines are the present MCSM calculations with the same color code. (c) $B\left(E 2 ; 0_{1}^{+} \rightarrow 2_{1}^{+}\right)$values from experiment [22] and by the present calculation.

prolate. We shall come to this point later. The correspondence between theoretical and experimental levels can be made with rather good agreement, including levels of higher spins.

Figure 3 depicts, for selected states of ${ }^{68,70,74,78} \mathrm{Ni}$ isotopes, potential energy surfaces (PESs) for the present Hamiltonian obtained by the constrained Hartree-Fock (CHF) method with the usual constraints on the quadrupole moments $Q_{0}$ and $Q_{2}$.

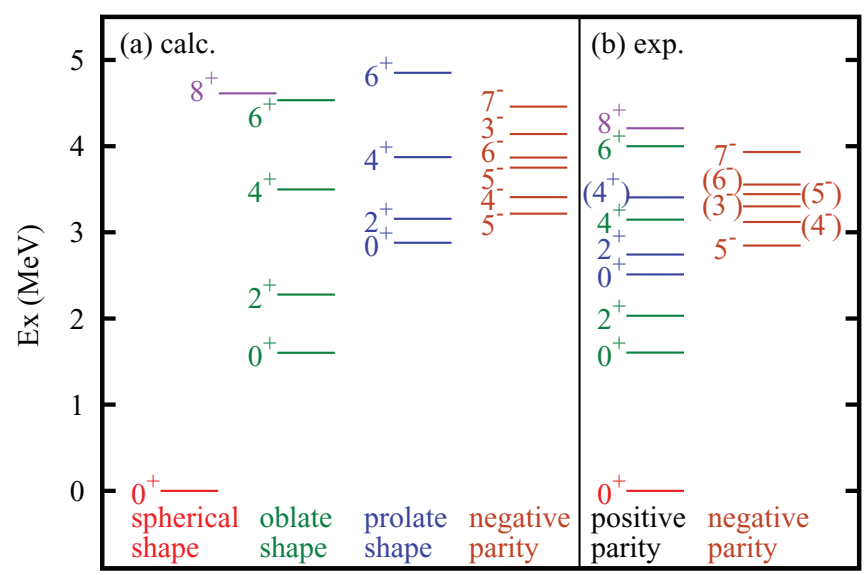

FIG. 2. (Color online) Energy levels of ${ }^{68} \mathrm{Ni}$ by (a) present calculation and (b) experiment [19-21].
We can see many features; for instance, for ${ }^{68} \mathrm{Ni}$, there is a spherical minimum stretched towards modest oblate region, as well as a prolate local minimum.

The MCSM wave function is expressed by a superposition of Slater determinants with the angular-momentum and parity projector $P\left[J^{\pi}\right]$,

$$
\Psi=\sum_{i} c_{i} P\left[J^{\pi}\right] \Phi_{i} .
$$

Here $c_{i}$ denotes an amplitude, and $\Phi_{i}$ stands for the Slater determinant consisting of one-nucleon wave functions $\phi_{1}^{(i)}, \phi_{2}^{(i)}, \ldots, \phi_{n}^{(i)}$ with

$$
\phi_{k}^{(i)}=\sum_{l} D_{k, l}^{(i)} u_{l}
$$

where $u_{l}$ is the $l$ th single-particle state in the original model space in the $m$ scheme, and $D$ implies an amplitude determined by the MCSM process. $\Phi_{i}$ is the product of the proton and neutron sectors, with $n$ being the number of valence protons or neutrons.

For each $\Phi_{i}$, we take the following procedure. We calculate its quadrupole moment matrix and diagonalize it. Three axes are obtained with $Q_{0}$ and $Q_{2}$ values. We then place a circle on the PES at the point corresponding to these $Q_{0}$ and $Q_{2}$ values. The size (i.e., area) of the circle is set to be proportional to the overlap probability between $\Psi$ and the normalized $P\left[J^{\pi}\right] \Phi_{i}$. Thus, the location of the circle implies the intrinsic shape of $\Phi_{i}$, and its size the importance of it in the eigenstate, $\Psi$. Note that the states $P\left[J^{\pi}\right] \Phi_{i}(i=1,2, \ldots)$ are not orthogonal to each other, in general, but the distribution pattern of the circles provides a unique and clear message on the intrinsic shape of the shell-model eigenstate, as we shall see.

Figure 3(a) shows such circles for the ground state of ${ }^{68} \mathrm{Ni}$. We see many large circles near the spherical point, $Q_{0}=Q_{2}=$ 0 . In general, there can be many points close to one another partly because each circle represents a Slater determinant and a two-body interaction, particularly its pairing components, mixes different Slater determinants. Those Slater determinants should have similar shapes so that the mixing between them can occur. We also see notable spreading of the distribution of circles from the spherical point. This implies the extent of the shape fluctuation. The $0_{2}^{+}$state in Fig. 3(b) shows similar spreading but the locations are shifted to the moderately oblate region $\left(\beta_{2} \sim-0.2\right)$. Although there is no clear potential barrier between the spherical and oblate regions of the PES, the antisymmetrization pushes the $0_{2}^{+}$state away from the $0_{1}^{+}$ state. Figure 3(c) exhibits many circles in a profound prolate minimum with $Q_{0} \sim 200 \mathrm{fm}^{2}\left(\beta_{2} \sim 0.4\right)$. We emphasize that we can analyze, in this way, the intrinsic shape even for $0^{+}$ states without referring to $E 2$ properties.

Figures 3(d) and 3(e) show the same plots for the $2_{1,2}^{+}$states. The $2_{1}^{+}$state exhibits a pattern almost identical to that of the $\mathrm{O}_{2}^{+}$state, which suggests the formation of the modestly oblate band. Such striking similarity is found also between the $0_{3}^{+}$ and $2_{2}^{+}$states with a strong-prolate-band assignment. The band structure can be further verified by $E 2$ matrix elements and is presented in Fig. 2 including $4^{+}$and $6^{+}$members. We note 

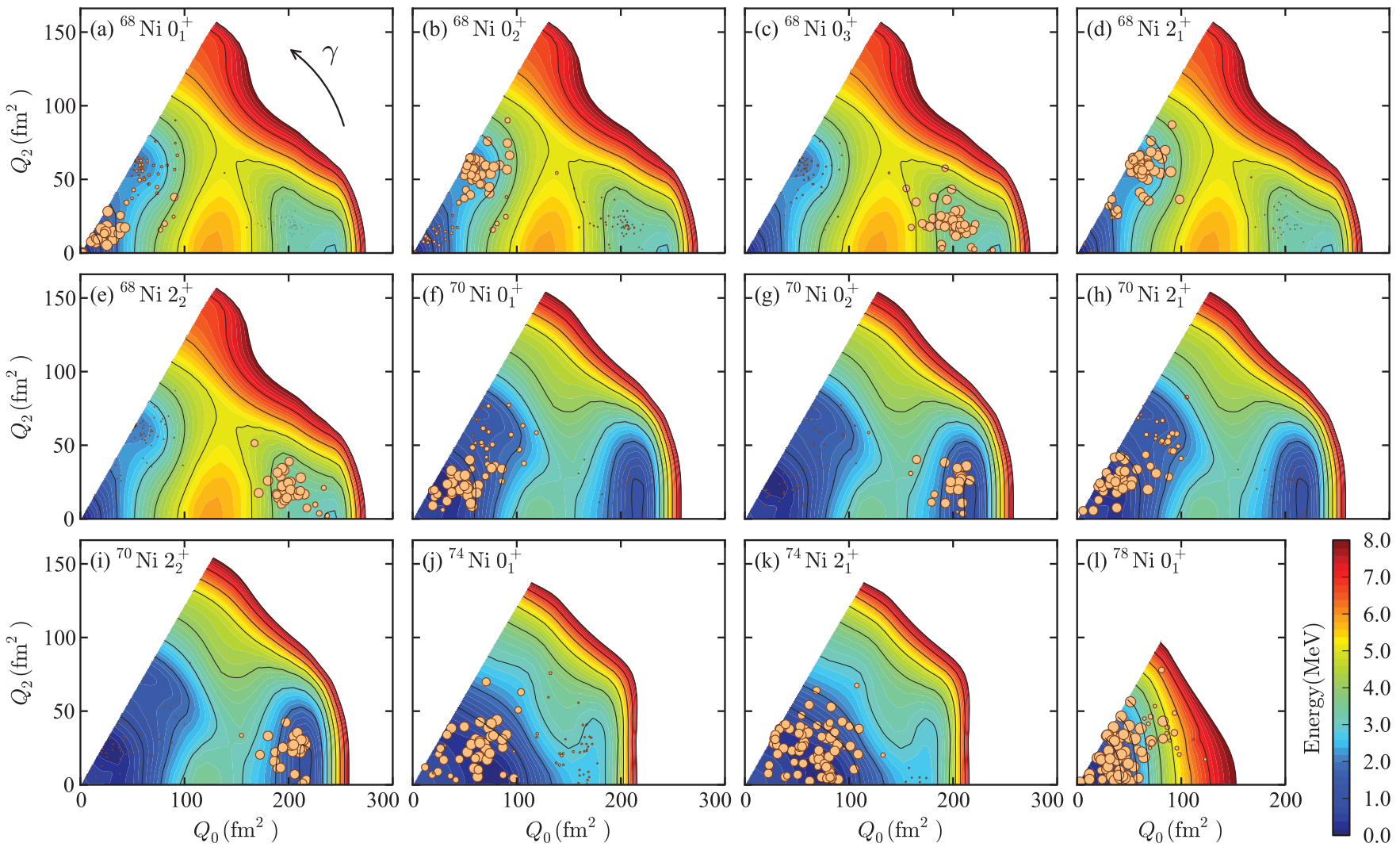

FIG. 3. (Color online) Potential energy surfaces (PESs) of Ni isotopes, coordinated by the usual $Q_{0}$ and $Q_{2}$ (or $\gamma$ ). The energy relative to the minimum is shown by contour plots. Circles on the PES represent shapes of MCSM basis vectors (see the text).

that the $0_{3}^{+}$and $2_{2}^{+}$states of ${ }^{68} \mathrm{Ni}$ were reported to be strongly deformed with $\beta_{2} \sim 0.4$ in shell-model calculations in [32].

Figures 4(a) and 4(b) show occupation numbers of proton and neutron orbits, respectively, for the $0_{1,2,3}^{+}$states of ${ }^{68} \mathrm{Ni}$. One sees drastic changes between the $0_{1}^{+}$and $0_{3}^{+}$states for proton $f_{7 / 2}$ and neutron $g_{9 / 2}$, while some other orbits show also sizable changes. Such changes are due to particle-hole excitations: mainly proton excitations from $f_{7 / 2}$ to $f_{5 / 2}$ and $p_{3 / 2,1 / 2}$, and neutron excitations from $f_{5 / 2}$ and $p_{1 / 2}$ to $g_{9 / 2}$. Once such excitations occur, the state can be deformed towards an ellipsoidal shape and large deformation energy is gained predominantly from the proton-neutron quadrupole interaction. The configuration structure of the $0_{3}^{+}$state seems to be beyond the applicability of truncated shell-model calculations [35,36].

We next discuss effective single-particle energy (ESPE), obtained from the monopole component $H_{m}$ of the Hamiltonian (see, for instance, [4] for more details). $H_{m}$ is written in terms of the number operator $n_{j}$ of each orbit $j$ (proton or neutron is omitted). The ESPE is calculated usually for configurations that are being filled, but we evaluate it for mixed configurations by a functional derivative $\epsilon_{j}=\left\langle\frac{\partial H_{m}}{\partial n_{j}}\right\rangle$ with the expectation values of $n_{j}$ 's for eigenstates being considered ${ }^{1}$. These $\epsilon_{j}$ 's are still spherical ESPEs, but are obtained with

${ }^{1}$ The contribution of identical particles in the same orbit becomes slightly different from the one by the filling scheme, but this difference is negligible in the present case. $\left\langle n_{j}\right\rangle$ of deformed states. From the viewpoint of the Nilsson model, $\epsilon_{j}$ 's correspond to Nilsson levels at the spherical limit, but the difference from the Nilsson model is that the $\epsilon_{j}$ 's vary as the deformation changes, due to the orbit dependence of the monopole component of nuclear forces. For protons, the ESPE of $f_{7 / 2}$ is increased by $\sim 2 \mathrm{MeV}$ in going from $0_{1}^{+}$to $0_{3}^{+}$ states, while ESPE of $f_{5 / 2}$ comes down by $\sim 1 \mathrm{MeV}$. Let us look into how these changes occur, based on the mechanism presented in $[4,8]$ : Because $g_{9 / 2}$ and $f_{7 / 2}$ are of $j_{>}(=l+1 / 2)$ type and $f_{5 / 2}$ is of $j_{<}(=l-1 / 2)$ type, the $g_{9 / 2^{-}} f_{7 / 2}\left(g_{9 / 2^{-}}\right.$ $f_{5 / 2}$ ) monopole interaction from the tensor force is repulsive (attractive). More neutrons in $g_{9 / 2}$ in the $\mathrm{O}_{3}^{+}$state result in the raising of the proton $f_{7 / 2}$ and the lowering of the proton $f_{5 / 2}$. Similarly, neutron holes in $f_{5 / 2}$ lead to the weakening of the attractive (repulsive) effect on the proton $f_{7 / 2}\left(f_{5 / 2}\right)$. All these effects reduce coherently the proton $f_{7 / 2}-f_{5 / 2}$ gap (i.e., the difference of the ESPEs of these orbits), making it $\sim 3 \mathrm{MeV}$ narrower in the $0_{3}^{+}$state, including other minor effects.

If a relevant shell gap becomes smaller, more particlehole excitations occur over this gap, leading to stronger deformation with more energy gain as mentioned above. A stronger deformation enhances particular configurations, for instance, more neutrons in $g_{9 / 2}$, which reduce the proton $f_{7 / 2}-f_{5 / 2}$ gap further. Thus, the change of the shell gap and strong deformation are interconnected in a self-consistent way. Figure 4(c) demonstrates this mechanism with an example of the proton $f_{7 / 2}-f_{5 / 2}$ gap obtained for the CHF wave function along the $\gamma=0^{\circ}$ and $60^{\circ}$ lines in Fig. 3, as a 

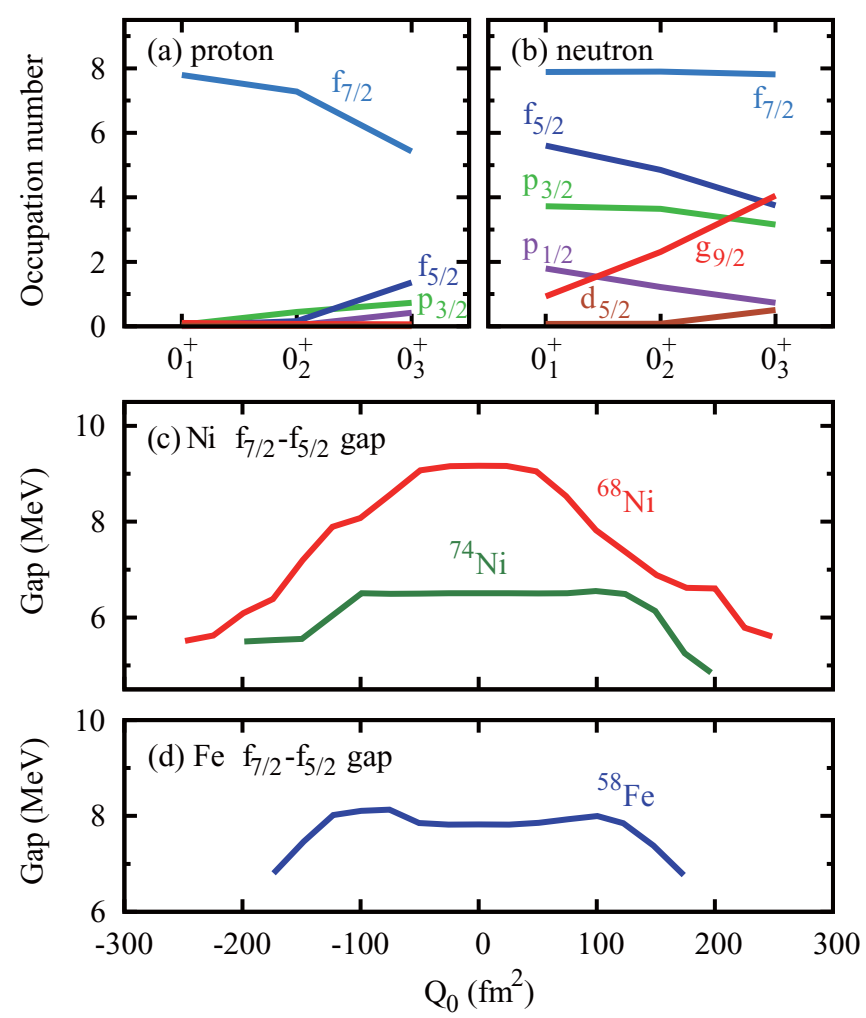

FIG. 4. (Color online) Occupation numbers for the $0^{+}$states of ${ }^{68} \mathrm{Ni}$ for (a) protons and (b) neutrons. Gap between $f_{7 / 2}$ and $f_{5 / 2}$ as a function of $Q_{0}$ of Fig. 3 for (c) ${ }^{68,74} \mathrm{Ni}$ and (d) ${ }^{58} \mathrm{Fe}$.

function of $Q_{0}$. For ${ }^{68} \mathrm{Ni}$, as $Q_{0}$ departs from zero, the gap remains large, producing a high barrier between spherical and deformed shapes. It, however, starts to come down beyond $\left|Q_{0}\right| \sim 50 \mathrm{fm}^{2}$, and is lowered by $3 \mathrm{MeV}$ for $\left|Q_{0}\right| \sim 200 \mathrm{fm}^{2}$. Although this is a consequence of strong deformation, it also enhances the deformation. Thus, the formation of a strongly prolate deformed band occurs by way of a non-linear mechanism, which stabilizes this band from spherical shape, by keeping the barrier high.

We now compare the phenomenon discussed above to the shell evolution which occurs in many cases as a function of $Z$ or $N[4,8,9]$. Figure 4(c) shows the proton $f_{7 / 2}-f_{5 / 2}$ gap of ${ }^{74} \mathrm{Ni}$ also. It starts with $\sim 6 \mathrm{MeV}$ at $Q_{0}=0$, much lower than the corresponding gap of ${ }^{68} \mathrm{Ni}$. This reduction of the gap is nothing but a consequence of the shell evolution with many neutrons occupying $g_{9 / 2}$. We shall call it Type I shell evolution hereafter. Correspondingly, phenomena like the reduction of the proton $f_{7 / 2}-f_{5 / 2}$ gap of ${ }^{68} \mathrm{Ni}$ at large deformation discussed above can then be called Type II shell evolution. Type I and II shell evolutions occur due to the occupation changes of single-particle orbits, while the changes are due to varying $Z$ and/or $N$ in Type I, but are due to particle-hole excitations within the same nucleus in Type II. The tensor force plays important roles in both cases as it is the primary origin of the change of the spin-orbit splitting.

Figure $4(\mathrm{c})$ shows that the $f_{7 / 2}-f_{5 / 2}$ gap of ${ }^{74} \mathrm{Ni}$ stays constant up to $\left|Q_{0}\right| \sim 120 \mathrm{fm}^{2}$, contrary to rapid change in ${ }^{68} \mathrm{Ni}$. Namely, Type II shell evolution is suppressed in
${ }^{74} \mathrm{Ni}$, as $g_{9 / 2}$ is occupied by many neutrons causing Type I shell evolution. Figure $3(\mathrm{j})$ shows the PES of ${ }^{74} \mathrm{Ni}$, where the profound local minimum of strong prolate deformation cannot be found consistently with the missing effect of Type II shell evolution. Such an interplay between Types I and II is of interest. The same gap is shown for ${ }^{58} \mathrm{Fe}$ in Fig. 4(d) as a typical standard case. The pattern is quite flat similar to ${ }^{74} \mathrm{Ni}$.

Type II shell evolution can thus be introduced as a mechanism not by the change of $Z$ or $N$, but by the change of major configurations. Type II shell evolution should enhance the appearance of the shape coexistence by stabilizing an isolated deformed local minimum. Further studies in various situations are of extreme interest. We stress that Type II shell evolution can occur in other nuclei provided that neutrons can be excited to a unique-parity orbit, reducing a proton spin-orbit splitting crucial for the deformation or vice versa. Some variations of this mechanism are also of great interest. The Type II shell-evolution mechanism should have been included in shell-model calculations in the past, for example [26,29,32], provided that a tensor-force component was included properly and a sufficiently large model space was taken. Nevertheless, its explicit recognition should help us to understand the underlying physics and foresee its impacts on structure issues in various regions of the nuclear chart including heavier and more exotic ones to be explored.

The prolate band being discussed comes down to the $0_{2}^{+}$and $2_{2}^{+}$states as $N$ increases from 40 to 42 or 44 [see Figs. $3(\mathrm{~g})$ and 3(i)]. The observed $2_{2}^{+}$level of ${ }^{70} \mathrm{Ni}$ is as low as $2 \mathrm{MeV}$, which is reproduced well by the present calculation, as shown in Fig. 1(b). The fact that this level has not been reproduced by calculations with limited configurations $[35,36]$ suggests that the $Z=28$ core is broken.

Moving to ${ }^{74} \mathrm{Ni}$, we observe that Figs. 3(j) and $3(\mathrm{k})$ exhibit another interesting pattern. The distribution of the circles becomes wide in both magnitude and $\gamma$ direction, i.e., triaxiality. A similar distribution is obtained also for the $2_{2}^{+}$ states, and the situation is the same for ${ }^{76} \mathrm{Ni}$. This pattern resembles the critical-point-symmetry $\mathrm{E}(5)$ [13].

Finally, we come to ${ }^{78} \mathrm{Ni}$, a doubly magic nucleus. Figure 3(1) shows the PES and wave function distribution. The PES has a spherical minimum which is very flat, as shown by the blue area of Fig. 3(1). One sees that the circles almost fill the entire flat area. This interesting pattern is seen also for the $2_{1}^{+}$state, reflecting a particular fluctuation. This fluctuation is much narrower in ${ }^{68} \mathrm{Ni}$, where the $E 2$ excitation from the ground state goes to very high $2^{+}$states, despite the low-lying $2_{1}^{+}$level. On the other hand, the overlap probabilities with the closed shell are $60 \%, 53 \%$, and $75 \%$ for ${ }^{56,68,78} \mathrm{Ni}$, respectively, similar to those in [37]. Thus, ${ }^{56,68,78} \mathrm{Ni}$ show very interesting variations regarding the appearance of the magicity, which deserve further investigation.

In summary, the advanced MCSM calculations present intriguing variations of shapes, analyzed in terms of intrinsic shapes. The shell evolution inside the same nucleus, called Type II shell evolution for clarification, can occur and can enhance shape coexistence. While Type I shell evolution appears in the $5 / 2^{-}-3 / 2^{-}$inversion of $\mathrm{Cu}$ isotopes $[9,38]$, 
not only particles in $g_{9 / 2}$ but also holes in $f_{5 / 2}$ contribute to the Type II case, making this case even more prominent. In addition, various shape evolutions are seen as $N$ changes, with notable fluctuations. Thus, the shapes of exotic nuclei provide us with many new features. In stable nuclei, the shape has often been discussed as functions of $N$ and $Z$. For instance, shape evolution from vibrational to rotational nuclei as $N$ increases. Such a simple classification may no longer be appropriate in exotic nuclei. Type II shell evolution is expected in heavier nuclei, as unique-parity orbits come down, and also because of the robust tensor-force effect $[9,39]$.

We thank Prof. B. R. Barrett for valuable comments. This work was in part supported by MEXT Grant-in-Aid for Scientific Research (A) 20244022. This work has been supported by HPCI (hp120284 and hp130024), and is a part of the RIKEN-CNS joint research project on large-scale nuclear-structure calculations. Y.T. acknowledges JSPS for support (No. 258994).
[1] M. G. Mayer, Phys. Rev. 75, 1969 (1949); O. Haxel, J. H. D. Jensen, and H. E. Suess, ibid. 75, 1766 (1949).

[2] J. Rainwater, Phys. Rev. 79, 432 (1950).

[3] A. Bohr and B. R. Mottelson, Nuclear Structure, Vol. II (Benjamin, New York, 1975).

[4] For a brief review, T. Otsuka, Phys. Scr. T 152, 014007 (2013).

[5] D. Steppenbeck et al., Nature 502, 207 (2013).

[6] T. Otsuka et al., Phys. Rev. Lett. 87, 082502 (2001).

[7] For reviews from the experimental side, A. Gade and T. Glasmacher, Prog. Part. Nucl. Phys. 60, 161 (2008); O. Sorlin and M.-G. Porquet, ibid. 61, 602 (2008).

[8] T. Otsuka, T. Suzuki, R. Fujimoto, H. Grawe, and Y. Akaishi, Phys. Rev. Lett. 95, 232502 (2005).

[9] T. Otsuka et al., Phys. Rev. Lett. 104, 012501 (2010).

[10] H. Morinaga, Phys. Rev. 101, 254 (1956).

[11] A. N. Andreyev et al., Nature 405, 430 (2000).

[12] K. Heyde and J. L. Wood, Rev. Mod. Phys. 83, 1467 (2011).

[13] F. Iachello, Phys. Rev. Lett. 85, 3580 (2000).

[14] N. Shimizu, T. Abe, Y. Tsunoda, Y. Utsuno, T. Yoshida, T. Mizusaki, M. Honma, and T. Otsuka, Prog. Theor. Exp. Phys. 01A205 (2012).

[15] N. Shimizu, Y. Utsuno, T. Mizusaki, T. Otsuka, T. Abe, and M. Honma, Phys. Rev. C 82, 061305(R) (2010).

[16] T. Otsuka, M. Honma, T. Mizusaki, N. Shimizu, and Y. Utsuno, Prog. Part. Nucl. Phys. 47, 319 (2001).

[17] Details available upon request to the corresponding authors.

[18] D. H. Gloeckner and R. D. Lawson, Phys. Lett. B 53, 313 (1974).

[19] Evaluated Nuclear Structure Data File (ENSDF), http://www.nndc.bnl.gov/ensdf/
[20] F. Recchia et al., Phys. Rev. C 88, 041302(R) (2013).

[21] S. Suchyta et al., Phys. Rev. C 89, 021301(R) (2014).

[22] B. Pritychenko, J. Choquette, M. Horoi, B. Karamy, and B. Singh, At. Data Nucl. Data Tables 98, 798 (2012).

[23] O. Perru et al., Phys. Rev. Lett. 96, 232501 (2006).

[24] N. Aoi et al., Phys. Lett. B 692, 302 (2010).

[25] K. Kaneko, M. Hasegawa, T. Mizusaki, and Y. Sun, Phys. Rev. C 74, 024321 (2006).

[26] S. M. Lenzi, F. Nowacki, A. Poves, and K. Sieja, Phys. Rev. C 82, 054301 (2010) and references therein.

[27] M. Girod, P. Dessagne, M. Bernas, M. Langevin, F. Pougheon, and P. Roussel, Phys. Rev. C 37, 2600 (1988).

[28] T. Ishii et al., Phys. Rev. Lett. 84, 39 (2000).

[29] O. Sorlin et al., Phys. Rev. Lett. 88, 092501 (2002).

[30] K. Langanke, J. Terasaki, F. Nowacki, D. J. Dean, and W. Nazarewicz, Phys. Rev. C 67, 044314 (2003).

[31] D. Pauwels, J. L. Wood, K. Heyde, M. Huyse, R. Julin, and P. Van Duppen, Phys. Rev. C 82, 027304 (2010).

[32] A. Dijon et al., Phys. Rev. C 85, 031301(R) (2012).

[33] C. J. Chiara et al., Phys. Rev. C 86, 041304(R) (2012).

[34] R. Broda et al., Phys. Rev. C 86, 064312 (2012) and references therein.

[35] M. Honma, T. Otsuka, T. Mizusaki, and M. Hjorth-Jensen, Phys. Rev. C 80, 064323 (2009).

[36] B. Cheal et al., Phys. Rev. Lett. 104, 252502 (2010).

[37] K. Sieja and F. Nowacki, Phys. Rev. C 85, 051301(R) (2012).

[38] K. T. Flanagan et al., Phys. Rev. Lett. 103, 142501 (2009).

[39] N. Tsunoda, T. Otsuka, K. Tsukiyama, and M. Hjorth-Jensen, Phys. Rev. C 84, 044322 (2011). 